\title{
Collision model for vehicle motion prediction after light impacts
}

\author{
Jing Zhou ${ }^{\mathrm{a}}$, Huei Peng ${ }^{\mathrm{b}} *$ and Jianbo Lu ${ }^{\mathrm{c}}$ \\ ${ }^{a}$ Department of Mechanical Engineering, University of Michigan, Ann Arbor, MI, USA; ${ }^{b}$ G036 Lay \\ Automotive Laboratory, Department of Mechanical Engineering, University of Michigan, Ann Arbor, \\ MI, USA; ${ }^{c}$ Research/Advanced Engineering, Ford Motor Company, USA
}

(Received 13 July 2007; final version received 21 December 2007)

\begin{abstract}
This article presents a collision model that predicts vehicle motions after a light impact. The focus of this work is on the characterisation of changes in vehicle kinematic states due to the impact; hence detailed analysis of vehicle component deformations is disregarded. Colliding vehicles are each modelled as rigid bodies with four degrees of freedom (longitudinal, lateral, yaw, and roll), which is different from the approach commonly used in the literature. The energy loss during impacts is accounted for through an empirical coefficient of restitution. In contrast to the conventional momentum-conservation-based method, the proposed approach takes tyre forces into account. Improved model prediction accuracy is demonstrated through numerical examples. The developed collision model is useful for the prediction of post-impact vehicle dynamics and the development of enhanced vehicle safety systems.
\end{abstract}

Keywords: vehicle collision model; 4-DOF vehicle dynamics model; post-impact vehicle states

\section{Introduction}

Vehicle collision mechanics are useful in fields such as vehicle crashworthiness, passenger injury prediction, and forensic accident reconstructions. Structural analysis methods can be used to construct complex numerical models (e.g., LS-DYNA [1] and PAM-CRASH [2]) to determine vehicle structural deformations after crashes. To use these models, a large set of vehicle component and material properties must be provided. Dedicated softwares such as HVE from the Engineering Dynamics Corporation [3] and PC-Crash from DSD GmbH [4] have been developed for accidents reconstruction purposes. The collision model in HVE is based on the EDSMAC simulation program, which evolved from the Simulation Model of Automobile Collisions (SMAC) program developed by Calspan [5] in the 1970s. To determine vehicle motions during a collision, the algorithm in HVE checks for possible deformation zones caused by the collision and calculates collision forces based on the vehicle structure stiffness and deflections. The forces are then used to calculate accelerations and velocities [3]. In contrast, PC-Crash uses a momentum-based 2- or 3-dimensional collision model. Energy

*Corresponding author. Email: hpeng@umich.edu

ISSN 0042-3114 print/ISSN 1744-5159 online

(C) 2008 Taylor \& Francis

DOI: $10.1080 / 00423110701882256$

http://www.informaworld.com 
loss is accounted for with a coefficient of restitution. It does not attempt to solve the collision forces during an impact. Instead, it relies on the law of momentum conservation to solve for velocity changes before and after the collision [4].

The focus of this study is on the characterisation of changes in vehicle kinematic states due to light impacts, and here light impacts refer to those collisions in which vehicles' structural deformations are not substantial, and dimensional changes can be ignored. Newton's equations of motion relating momentum with impulse are the foundations for vehicle collision mechanics [4-9]. Brach uses this approach in his books [6,7] to model collision events. The vehicle is treated as a rigid body with three degrees of freedom (DOF), and vehicle deformations and contact forces are not directly modelled. Another common assumption made in earlier treatments is that other external forces, such as tyre forces and aerodynamic drag, are negligible. Therefore, linear and angular momentums are conserved for the two-vehicle system. Although in many situations the tyre forces are much smaller than the impact forces, the momentum change induced by tyre forces can be substantial during an impact, especially when tyre slip angles are large. As will be shown later in this paper, if tyre forces are ignored, significant deviations can be introduced in predicting lateral motions of the vehicle.

Another novel idea proposed in this paper is to model colliding vehicles as rigid bodies with four DOF, as opposed to bodies confined in the $x-y$ plane. This approach makes it possible to predict post-impact vehicle roll motion. This additional DOF, along with the inclusion of tyre forces during the collision, reduces the error in predicting vehicle lateral and yaw motions. The proposed model is not intended to capture the detailed structural deformations; instead, we are more concerned with the changes in vehicle motions, in particular, longitudinal and lateral velocities as well as yaw and roll rates. Simulation results will be demonstrated by comparing the prediction results of this 4-DOF model against the commercial vehicle dynamics software $\mathrm{CarSim}^{\mathrm{TM}}$.

The rest of this paper is organised as follows. The momentum-based collision model is reviewed in Section 2. Section 3 presents a new 4-DOF vehicle dynamics model that accounts for both impact and lateral tyre forces. The formulation of the inter-vehicle collision problem using the 4-DOF model is detailed in Section 4. Section 5 compares the computation results of the proposed approach against those provided by the traditional momentum-conservation method. Finally, conclusions are drawn in Section 6.

\section{Momentum-conservation-based collision model}

A well-known impact model based on the conservation of momentum method was developed by Brach [6,7], which has been used extensively for accident reconstruction. This planar-model is reviewed in this section, which serves as both the benchmark, and the foundation for our new model. Figure 1 shows a planar view of the free body diagrams of two colliding vehicles. Throughout the remainder of this paper, the target vehicle is denoted as Vehicle 1, and the bullet vehicle is denoted as Vehicle 2. An earth-fixed coordinate system $(X O Y)$ is assumed to align with the road tangential direction. The orientation angle of the vehicles is denoted as $\theta$. An additional local coordinate system $(n-t)$ is associated with the impact impulse. The $t$-axis is parallel to an imagined crush plane common to both vehicles, and the $n$-axis is normal to that plane. The choice of the crush plane is case-dependent and should define a nominal deformation surface. The $n-t$ coordinate system is related to the $X O Y$ coordinate system through the angle $\Gamma$.

To make the collision problem manageable, additional assumptions need to be made. The resultant impulse vector is assumed to have a specific point of application. Following Brach 


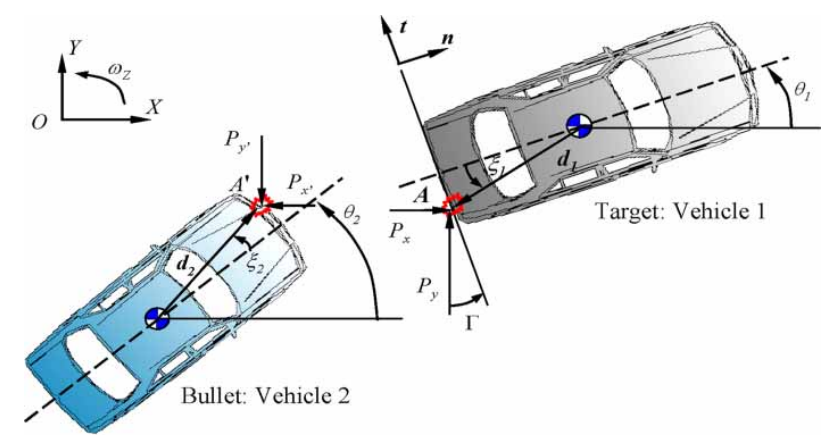

Figure 1. A planar view of the free body diagrams for colliding vehicles.

[6], it is assumed that the location of this point $\left(A / A^{\prime}\right)$ is known and can be located by a distance $(d)$ and a polar angle $(\xi)$ measured from the vehicle centre of gravity (CG).

Since the vehicles are confined to the $x-y$ plane, six pre-impact vehicle kinematic states $\left(v_{1 x}, v_{1 y}, \omega_{1 z}, v_{2 x}, v_{2 y}, \omega_{2 z}\right)$ are sufficient to describe the motions of the two vehicles. The values of these variables are assumed to be known. Accordingly, there are six unknown post-impact vehicle motion variables $\left(V_{1 x}, V_{1 y}, \Omega_{1 z}, V_{2 x}, V_{2 y}, \Omega_{2 z}\right)$ to be calculated. Six independent equations are sought to solve these variables.

In ref. [6], tyre forces are ignored and only collision-induced impulse inputs are considered. Linear momentum is thus conserved for the two-vehicle system:

$$
\begin{aligned}
& P_{x}=m_{1} \cdot\left(V_{1 x}-v_{1 x}\right)=-m_{2} \cdot\left(V_{2 x}-v_{2 x}\right) \\
& P_{y}=m_{1} \cdot\left(V_{1 y}-v_{1 y}\right)=-m_{2} \cdot\left(V_{2 y}-v_{2 y}\right)
\end{aligned}
$$

By taking moment of the momentum about vehicle CGs, two additional equations can be obtained to relate pre- and post-impact vehicle yaw rates:

$$
\begin{aligned}
& I_{z z 1}\left(\Omega_{1 z}-\omega_{1 z}\right)=P_{x} d_{c}-P_{y} d_{d} \\
& I_{z z 2}\left(\Omega_{2 z}-\omega_{2 z}\right)=P_{x} d_{a}-P_{y} d_{b}
\end{aligned}
$$

where $d_{a}=d_{2} \sin \left(\theta_{2}+\xi_{2}\right), d_{b}=d_{2} \cos \left(\theta_{2}+\xi_{2}\right), d_{c}=d_{1} \sin \left(\theta_{1}+\xi_{1}\right), d_{d}=d_{1} \cos \left(\theta_{1}+\xi_{1}\right)$.

Finally, two more equations are derived from collision constraints: the coefficient of restitution and the coefficient of tangential interaction [6]. The coefficient of restitution $(e)$ is a lumped measure of the energy loss during an impact. It is defined as the negative ratio of the final to initial relative normal velocity components at the impact point $\left(A / A^{\prime}\right)$.

$$
e=-\frac{V_{2 n}-V_{1 n}}{v_{2 n}-v_{1 n}}
$$

The magnitude of the coefficient of restitution depends on the body/bumper materials, surface geometry [10], impact velocity [11], and so on. Determining its value accurately requires extensive empirical data. Typical values of $e$ are found to have an inverse relationship to closing velocity [12], and range between 0 and 0.3 for rear-end impacts [13]. As shown in Figure 2, large variation in test results exists. Inter-vehicle velocity difference also significantly affects its value. 


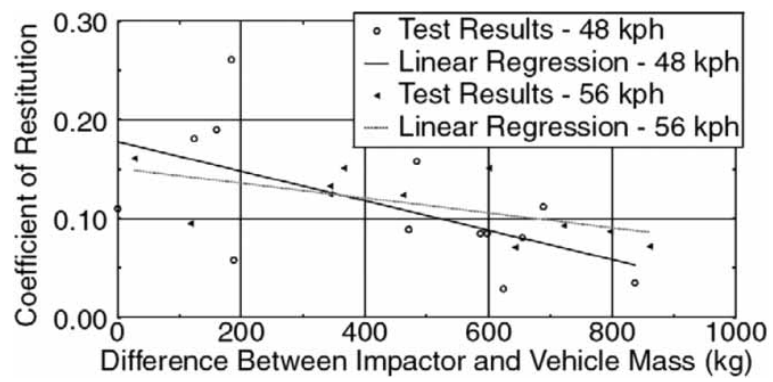

Figure 2. Coefficient of restitution versus mass difference in rear collision tests [13].

From Figure 1, the normal components of the vehicle velocities at the impact point $\left(A / A^{\prime}\right)$ can be substituted into Equation (5) to obtain

$$
\begin{aligned}
& \left(V_{1 y}-d_{d} \Omega_{1 z}-V_{2 y}-d_{b} \Omega_{2 z}\right) \sin \Gamma+\left(V_{1 x}+d_{c} \Omega_{1 z}-V_{2 x}+d_{a} \Omega_{2 z}\right) \cos \Gamma \\
& \quad=-e\left[\left(v_{1 y}-d_{d} \omega_{1 z}-v_{2 y}-d_{b} \omega_{2 z}\right) \sin \Gamma+\left(v_{1 x}+d_{c} \omega_{1 z}-v_{2 x}+d_{a} \omega_{2 z}\right) \cos \Gamma\right]
\end{aligned}
$$

The coefficient of tangential interaction $(\mu)$ is a lumped measure of the frictional dissipation during the impact, and relates the tangential impulse with the normal impulse:

$$
\mu=\frac{P_{t}}{P_{n}}
$$

A detailed discussion of inter-vehicle friction and its application in accident reconstruction can be found in ref. [14]. By decomposing the total impulse along the $n-t$ axes, Equation (7) becomes

$$
\mu \cdot\left(P_{x} \cos \Gamma+P_{y} \sin \Gamma\right)=P_{y} \cos \Gamma-P_{x} \sin \Gamma
$$

Collecting Equations (1-4), (6), (8), and assembling them into a matrix form lead to

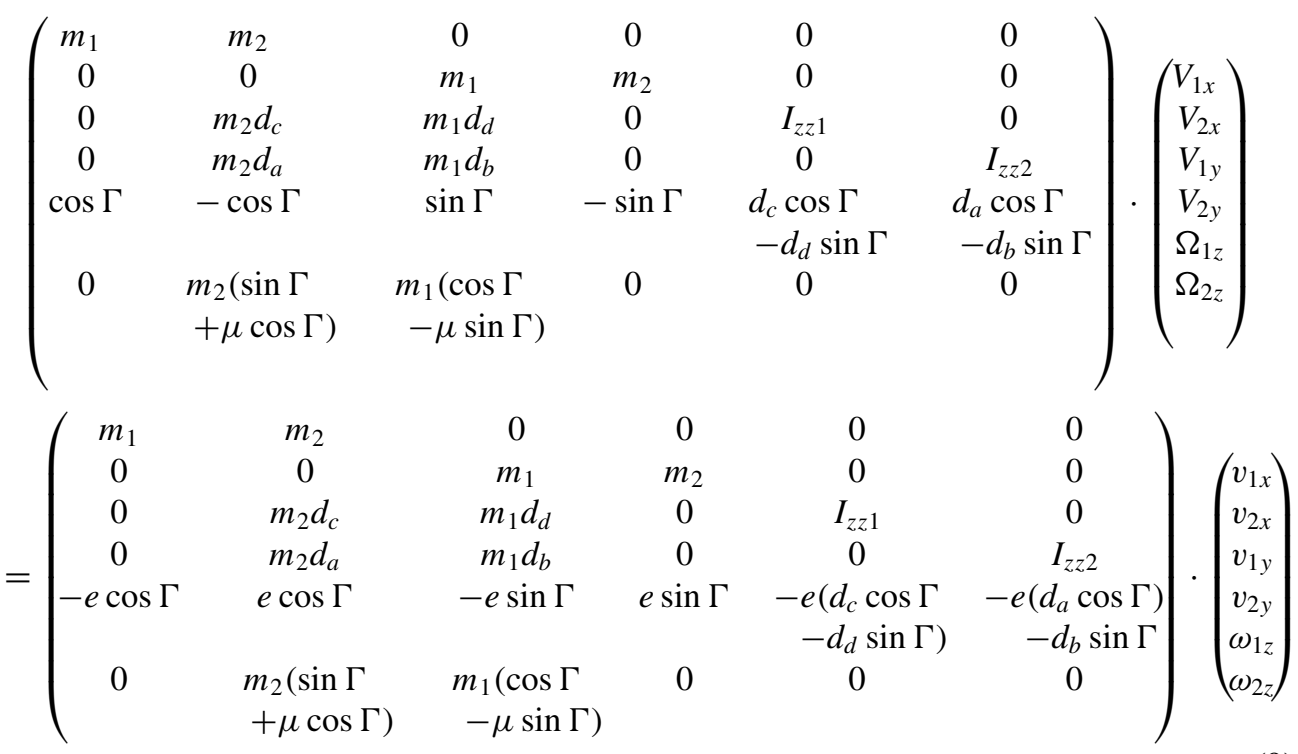

If vehicle parameters and collision conditions (such as orientation angle, coefficient of restitution, etc.) are known, Equation (9) can be solved either forwards (given the pre-impact states, 
to solve the post-impact states) or backwards (given the post-impact states, to reconstruct the pre-impact states). The only exception is when $e=0$, the coefficient matrix on the right-hand side becomes singular, and the pre-impact vehicle states cannot be uniquely determined.

\section{Four-DOF vehicle dynamics model}

The impact model presented in the previous section is a 3-DOF planar model, which only accounts for longitudinal, lateral, and yaw motions. The model in PC-Crash package [4] is 3-dimensional, and it does allow the impact to influence the 6-DOF motion of a rigid body. However, the assumption that ground forces can be completely neglected during the collision phase is reasonable for spacecraft collisions, but not for ground vehicle collisions. In addition, the impact-induced roll motion cannot be captured by the planar model.

In this section, a 4-DOF model based on Segel's lateral-yaw-roll model [15] is used to develop a new impact model. Heave and pitch motions of the vehicle are ignored. The schematic diagram of the vehicle model is shown in Figure 3.

This vehicle model separates the rolling (sprung) mass $m_{R}$ from the non-rolling (unsprung) mass $m_{\mathrm{NR}}$. The rolling mass interacts with the non-rolling mass via suspensions (not shown). The effect of the suspension elements at four corners is lumped into an equivalent torsional spring and a damper around the roll axis (see also Figure 4c). The overall CG of the vehicle is denoted $M$. The coordinate system $x y z$ is fixed on the vehicle body, and its orientation conforms to the ISO coordinate convention. The roll axis (the same as the $x$-axis here) passes through the non-rolling mass and is assumed to be parallel to the ground. The distance between the rolling mass CG and the roll axis is denoted $h$, whereas the height of the overall CG above the ground is denoted $h_{\mathrm{CG}}$.

Figure 4 shows this vehicle model in three orthogonal views with impact forces applied to the rear bumper. Also shown are vehicle longitudinal velocity $\left(v_{x}\right)$, lateral velocity $\left(v_{y}\right)$, yaw rate $\left(\omega_{z}\right)$, and roll rate $\left(\omega_{x}\right)$. The impact forces $\left(F_{x}, F_{y}\right)$ are assumed to be horizontal only. The impact position $(A)$ is located by the coordinates $\left(x_{A}, y_{A}, z_{A}\right)$, and both $x_{A}$ and $y_{A}$ are negative in the rear-end collision scenario depicted in Figure 4. The dynamic equations of motion in terms of longitudinal, lateral velocities, as well as rotational motion about the $x$-axis (roll) and the $z$-axis (yaw) can be written as

$$
\begin{gathered}
M\left(\dot{v}_{x}-v_{y} \omega_{z}\right)=F_{x} \\
M\left(\dot{v}_{y}+v_{x} \omega_{z}\right)-m_{R} h \dot{\omega}_{x}=F_{y}+F_{y f}+F_{y r}
\end{gathered}
$$

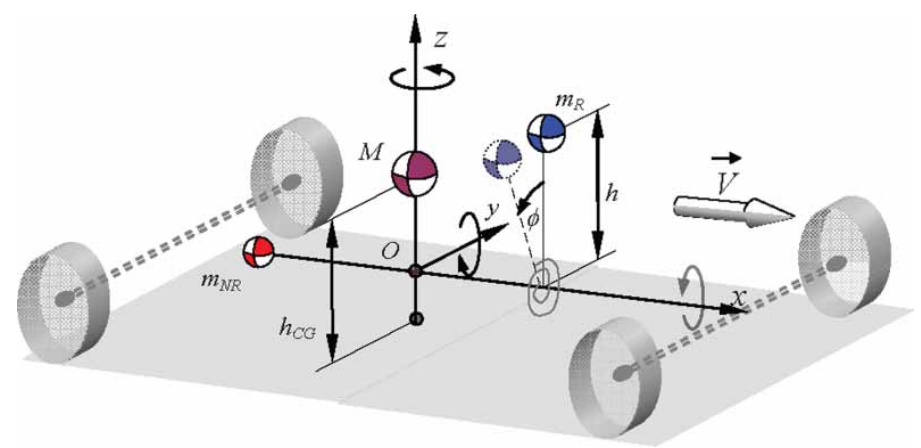

Figure 3. 3D schematic diagram of a 4-DOF vehicle model. 


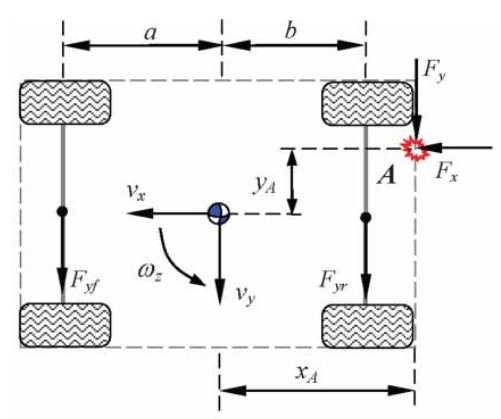

(a) Top view

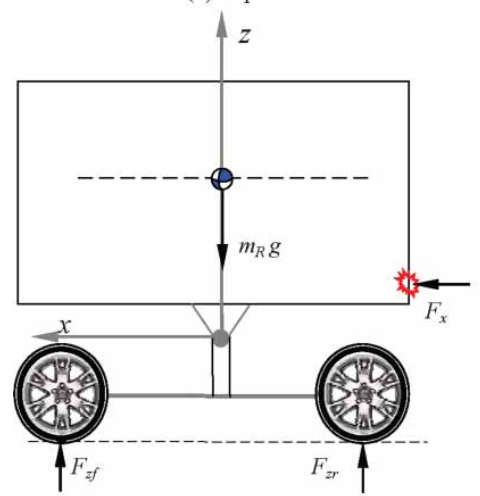

(b) Side view

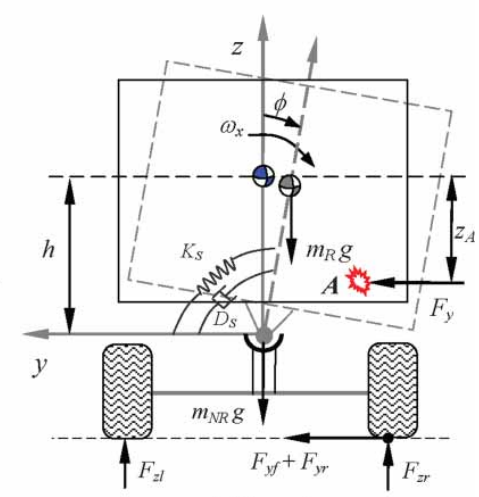

(c) Rear view

Figure 4. Schematic diagrams of the vehicle model with impact forces applied.

$$
\begin{gathered}
I_{z z} \dot{\omega}_{z}+I_{x z} \dot{\omega}_{x}=x_{A} F_{y}-y_{A} F_{x}+a F_{y f}-b F_{y r} \\
I_{x x s} \dot{\omega}_{x}+I_{x z} \dot{\omega}_{z}-m_{R} h\left(\dot{v}_{y}+v_{x} \omega_{z}\right)=F_{y}\left(z_{A}-h\right)+\left(m_{R} g h-K_{s}\right) \phi-D_{s} \omega_{x}
\end{gathered}
$$

where $\begin{cases}F_{y f}=C_{f}\left(\delta_{f}-\frac{v_{y}+a \omega_{z}}{v_{x}}\right), & \left|F_{y f}\right|<M g \mu_{R} \frac{b}{L} . \\ F_{y r}=C_{r}\left(\frac{-v_{y}+b \omega_{z}}{v_{x}}\right), & \left|F_{y r}\right|<M g \mu_{R} \frac{a}{L}\end{cases}$

In other words, the lateral tyre forces $F_{y f}$ and $F_{y r}$ are assumed to be linear with slip angles, but saturate at certain tyre adhesion limits.

\section{Four-DOF vehicle collision model}

In this section, a 4-DOF vehicle impact model is developed. To simplify the equations, it is further assumed that the fore-aft centreline of the target vehicle is parallel to the road $\left(\theta_{1}=0\right)$. Two additional local coordinate systems are defined in Figure 5. The coordinate system $x-y$ is fixed on the target vehicle and the system $x^{\prime}-y^{\prime}$ is fixed on the bullet vehicle, corresponding to their longitudinal and lateral axes respectively. The action and reaction impact forces can then be decomposed into $F_{x}$ and $F_{y}$, or $F_{x^{\prime}}$ and $F_{y^{\prime}}$, which relates more directly to the vehicle dynamics. A total of 12 unknowns need to be solved: post-impact longitudinal and lateral velocities, yaw and roll rates for both bullet and target vehicles $\left(V_{1 x}, V_{1 y}, \Omega_{1 z}, \Omega_{1 x}, V_{2 x^{\prime}}, V_{2 y^{\prime}}, \Omega_{2 z}, \Omega_{2 x^{\prime}}\right)$, as well as the collision-induced impulses acting on the vehicles $\left(P_{x}, P_{y}, P_{x^{\prime}}, P_{y^{\prime}}\right)$. The eight pre-impact vehicle states $\left(v_{1 x}, v_{1 y}, \omega_{1 z}, \omega_{1 x}, v_{2 x^{\prime}}, v_{2 y^{\prime}}, \omega_{2 z}, \omega_{2 x^{\prime}}\right)$ are assumed to be available. 

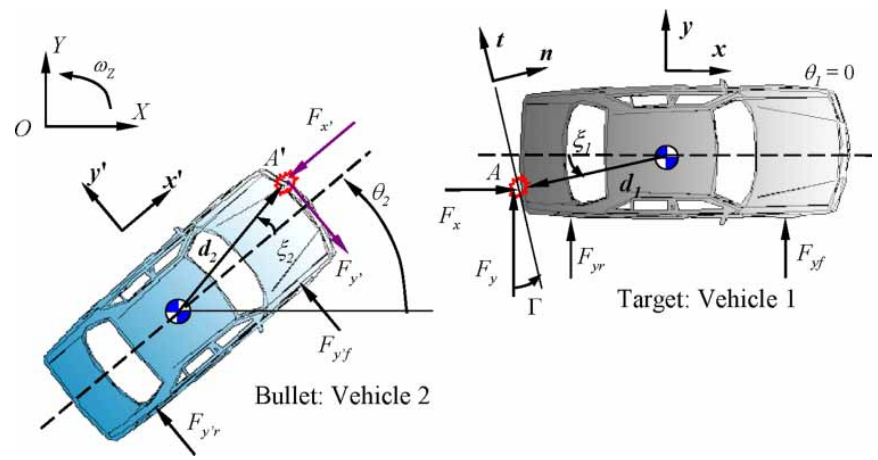

Figure 5. A planar view of colliding vehicles with body-fixed coordinates systems defined.

Within the short duration of a collision, acceleration levels are high, velocity changes are limited in magnitude, and displacements are negligible. The typical time duration for a collision is around $0.1-0.2 \mathrm{~s}[12,16,17]$. A time duration of this magnitude justifies a trapezoidal approximation for the integral of cross-terms in Equations (10)-(13). Equations (14)-(17) present the formulation in integral form for the target vehicle. The corresponding four equations for the bullet vehicle can be obtained in the same way and are omitted here.

$$
\begin{aligned}
& M_{1} \cdot\left(V_{1 x}-v_{1 x}\right)-M_{1} \frac{\Delta t}{2}\left(V_{1 y} \Omega_{1 z}+v_{1 y} \omega_{1 z}\right)=P_{x} \\
& M_{1} \cdot\left(V_{1 y}-v_{1 y}\right)+M_{1} \frac{\Delta t}{2}\left(V_{1 x} \Omega_{1 z}+v_{1 x} \omega_{1 z}\right)-m_{R 1} h_{1} \cdot\left(\Omega_{1 x}-\omega_{1 x}\right) \\
& =P_{y}-\frac{\Delta t}{2} C_{f 1}\left(\frac{V_{1 y}+a_{1} \Omega_{1 z}}{V_{1 x}}-\frac{v_{1 y}+a_{1} \omega_{1 z}}{v_{1 x}}\right) \\
& \quad-\frac{\Delta t}{2} C_{r 1}\left(\frac{V_{1 y}-b_{1} \Omega_{1 z}}{V_{1 x}}-\frac{v_{1 y}-b_{1} \omega_{1 z}}{v_{1 x}}\right) \\
& \quad \quad-\frac{\Delta t}{2} b_{1} C_{r 1}\left(\frac{V_{1 y}-b_{1} \Omega_{1 z}}{V_{1 x}}-\frac{v_{1 y}-b_{1} \omega_{1 z}}{v_{1 x}}\right) \\
& I_{z z 1}\left(\Omega_{1 z}-\omega_{1 z}\right)+I_{x z 1}\left(\Omega_{1 x}-\omega_{1 x}\right)=P_{y} x_{A}-P_{x} y_{A} \\
& I_{x x s 1}\left(\Omega_{1 x}-\omega_{1 x}\right)+I_{x z 1}\left(\Omega_{1 z}-\omega_{1 z}\right)-m_{R 1} h_{1} \cdot\left(V_{1 y}-v_{1 y}\right)-m_{R 1} h_{1} \frac{\Delta t}{2}\left(V_{1 x} \Omega_{1 z}+v_{1 x} \omega_{1 z}\right) \\
& =P_{y}\left(z_{A}-h_{1}\right)-D_{s 1} \frac{\Delta t}{2}\left(\Omega_{1 x}+\omega_{1 x}\right)
\end{aligned}
$$

In the above equations, the duration of collision $(\Delta t)$ is assumed to be known. Lateral tyre forces appear in the equations for lateral and yaw motions. When the vehicle is subject to substantial lateral/yaw motions, lateral tyre forces may reach saturation limits even before the collision ends. In that case, the lateral tyre force terms in Equations (15) and (16) will be replaced with tyre adhesion limits.

Two additional equations are derived from the coefficient of restitution $(e)$ and the coefficient of tangential interaction $(\mu)$, both of which are assumed to be known a priori. The restitution relationship is described by Equation (18) and the tangential interaction is accounted for in 
the same way as in Equation (8).

$$
\begin{aligned}
& {\left[\left(V_{2 x^{\prime}} \cos \theta_{2}-V_{2 y^{\prime}} \sin \theta_{2}-d_{a} \Omega_{2 z}\right) \cos \Gamma+\left(V_{2 x^{\prime}} \sin \theta_{2}+V_{2 y^{\prime}} \cos \theta_{2}+d_{b} \Omega_{2 z}\right) \sin \Gamma\right]} \\
& \quad-\left[\left(V_{1 x}+d_{c} \Omega_{1 z}\right) \cos \Gamma+\left(V_{1 y}-d_{d} \Omega_{1 z}\right) \sin \Gamma\right]=e\left[\left(v_{1 x}+d_{c} \omega_{1 z}\right) \cos \Gamma\right. \\
& \left.\quad+\left(v_{1 y}-d_{d} \omega_{1 z}\right) \sin \Gamma\right] \\
& \quad-e\left[\left(v_{2 x^{\prime}} \cos \theta_{2}-v_{2 y^{\prime}} \sin \theta_{2}-d_{a} \omega_{2 z}\right) \cos \Gamma+\left(v_{2 x^{\prime}} \sin \theta_{2}+v_{2 y^{\prime}} \cos \theta_{2}+d_{b} \omega_{2 z}\right) \sin \Gamma\right]
\end{aligned}
$$

Finally, two more equations project the collision impulses from the bullet vehicle coordinate frame to the target vehicle coordinate frame.

$$
\begin{aligned}
& P_{x}=-P_{x^{\prime}} \cos \theta_{2}+P_{y^{\prime}} \sin \theta_{2} \\
& P_{y}=-P_{x^{\prime}} \sin \theta_{2}-P_{y^{\prime}} \cos \theta_{2}
\end{aligned}
$$

The 12 equations can be collected and assembled in a matrix form that relates the post-impact vehicle states to the pre-impact states. Equation (21) presents the block-matrix formulation for a special collision scenario, in which both vehicles are assumed to travel along their own longitudinal axes when the collision occurs. In other words, before the impact, $v_{y}, \omega_{z}, \omega_{x}$ are all zero for both vehicles. The specific terms in matrix $\mathbf{A}$ and vector $\mathbf{B}$ are detailed in the Appendix. The formulation for cases with non-zero pre-impact $v_{y}, \omega_{z}, \omega_{x}$ can be readily accommodated by modifying corresponding entries in $\mathbf{B}$.

$$
\left(\begin{array}{ccc}
A_{11} & \mathbf{0} & A_{13} \\
\mathbf{0} & A_{22} & A_{23} \\
A_{31} & A_{32} & A_{33}
\end{array}\right) \cdot \mathbf{x}=\mathbf{B}
$$

where $\mathbf{x}=\left(\begin{array}{llllllllllll}V_{1 x} & V_{1 y} & \Omega_{1 z} & \Omega_{1 x} & V_{2 x^{\prime}} & V_{2 y^{\prime}} & \Omega_{2 z} & \Omega_{2 x^{\prime}} & P_{x} & P_{y} & P_{x^{\prime}} & P_{y^{\prime}}\end{array}\right)^{\prime}$.

It should be pointed out that unknown velocities $\left(V_{1 x}, V_{1 y}, V_{2 x^{\prime}}, V_{2 y^{\prime}}\right)$ also appear in the coefficient matrix on the left-hand side, thus the 12 unknowns cannot be solved by direct matrix inversion. This problem can be cast into a non-linear least-squares formulation and be solved by general optimisation routines (such as lsqnonlin in Matlab). As a more practical solution, pre-impact velocities $\left(v_{1 x}, v_{1 y}, v_{2 x^{\prime}}, v_{2 y^{\prime}}\right)$ can be used as initial guesses for the four unknown velocities. Then the post-impact vehicle states and corresponding impulses can be obtained by iteratively solving the 12 algebraic equations, until a specified tolerance is met between two successive iterations.

The contact force at the impact point cannot be determined directly from this model. Various collision impulse shapes (sinusoidal, square, triangular, and sine square profiles) have been proposed to fit observed accelerometer signals in crash experiments $[17,18]$. In the end, after the collision impulses have been resolved, given the assumed collision time duration $\Delta t$, the impact force profile can be approximated.

\section{Simulation results}

The accuracy of the developed impact model is studied in this section. The computation results from a commercial vehicle dynamics software, CarSim [19], provide a benchmark to assess the accuracy of the planar and the proposed 4-DOF impact models.

In the simulation, both the bullet and target vehicles are of the same configuration. The vehicle parameters are summarised in Table 1, which corresponds to the 'baseline big SUV' 
Table 1. Vehicle parameters for the 4-DOF model.

\begin{tabular}{llcc}
\hline Parameter & \multicolumn{1}{c}{ Description } & Value & Unit \\
\hline$M$ & Total vehicle mass & 2450 & $\mathrm{~kg}$ \\
$m_{R}, m_{\mathrm{NR}}$ & Rolling mass, non-rolling mass & 2210,240 & $\mathrm{~kg}$ \\
$a, b$ & Distance from axles to vehicle CG & $1.105,1.745$ & $\mathrm{~m}$ \\
$h_{\mathrm{CG}}$ & CG height above the ground & 0.66 & $\mathrm{~m}$ \\
$h$ & Distance from sprung mass CG to the roll axis & 0.40 & $\mathrm{~m}$ \\
$I_{z z}$ & Vehicle yaw moment of inertia about $z$-axis & 4946 & $\mathrm{~kg} \cdot \mathrm{m}^{2}$ \\
$I_{x z}$ & Sprung mass product of inertia about roll and yaw axes & 40 & $\mathrm{~kg} \cdot \mathrm{m}^{2}$ \\
$I_{x x s}$ & Sprung mass roll moment of inertia about roll axis & 1597 & $\mathrm{~kg} \cdot \mathrm{m}^{2}$ \\
$K_{s}$ & Total suspension roll stiffness & 94000 & $\mathrm{~N} \cdot \mathrm{m} / \mathrm{rad}$ \\
$D_{s}$ & Total suspension roll damping & 8000 & $\mathrm{~N} \cdot \mathrm{m} \cdot \mathrm{s} / \mathrm{rad}$ \\
$C_{f}, C_{r}$ & Axle cornering stiffness, front and rear & 145750,104830 & $\mathrm{~N} / \mathrm{rad}$ \\
\hline
\end{tabular}

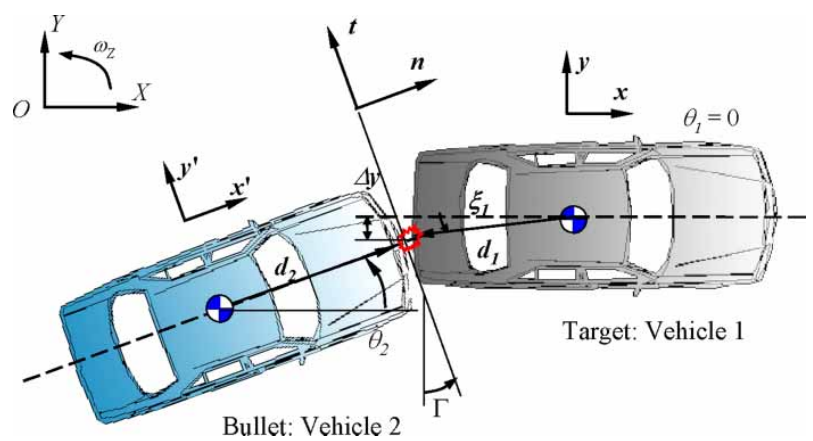

Figure 6. An angled rear-end collision. Assume the bullet vehicle is only subject to longitudinal forces.

dataset in CarSim. The collision scenario is illustrated in Figure 6. Before the impact, the target vehicle is aligned with the road tangent, whereas the bullet vehicle has a certain orientation angle $\theta_{2}$. Both the bullet and target vehicles are travelling along their longitudinal directions when the collision occurs, with $v_{1 x}=29 \mathrm{~m} / \mathrm{s}, v_{2 x^{\prime}}=33.5 \mathrm{~m} / \mathrm{s}$; their initial lateral velocity, yaw rate, and roll rate are all zero. The coefficient of restitution $(e)$ is assumed to be 0.20 for this rear-end crash. It is further assumed that no tangential impulse is generated during the collision $(\mu=0)$. The road adhesion condition is assumed to be at $\mu_{R}=0.70$.

The study will focus on the post-impact motions of the target vehicle. It is assumed that the impact point of the bullet vehicle is located at the centre of the front bumper $\left(\xi_{2}=0\right)$, and no lateral impulse is generated on the bullet vehicle $\left(P_{y^{\prime}}=0\right)$. Therefore, the bullet vehicle is subject only to longitudinal resistant impulses, and no post-impact lateral/yaw/roll motions will be generated.

The impact location of the target vehicle $(\Delta y)$ is $0.1 \mathrm{~m}$ to the left of the rear bumper centre, and the impact incidence angle $\theta_{2}$ is $25^{\circ}$. Computation results of more general settings will be presented later in this section. Based on the computation in CarSim, after the impact the bullet vehicle remains on the original course, but its velocity is reduced to $V_{2 x^{\prime}}=30.6 \mathrm{~m} / \mathrm{s}$. The postimpact states of the target vehicle at the precise moment the collision is over $(\Delta t=0.15 \mathrm{~s})$ are shown in Table 2 under the column heading 'CarSim.'

The time responses to impact forces of the target vehicle are shown in Figure 7. The collision starts at $2 \mathrm{~s}$ and ends at $2.15 \mathrm{~s}$. The impact forces $\left(F_{x}, F_{y}\right)$, which are based on the collision impulse, are assumed to follow triangle profiles. Intense yaw motion and transient roll motion are excited by the collision. As a result, large tyre slip angles make lateral tyre forces saturate at adhesion limits. Since no driver intervention or activation of vehicle stability systems is 
Table 2. Comparison of computation results in an angled rear-end collision.

\begin{tabular}{lccccr}
\hline \multirow{2}{*}{ Kinematic states } & & \multicolumn{3}{c}{ Target vehicle } \\
\cline { 3 - 6 } Pre-impact & & Bullet vehicle & CarSim & 4-DOF & Planar \\
& $v_{x}(\mathrm{~m} / \mathrm{s})$ & $33.5 \times \cos 25^{\circ}$ & & 29 & \\
& $v_{y}(\mathrm{~m} / \mathrm{s})$ & $33.5 \times \sin 25^{\circ}$ & & 0 & \\
\multirow{2}{*}{ Post-impact } & $\omega_{z}(\mathrm{deg} / \mathrm{s})$ & 0 & & 0 & 31.9 \\
& $V_{x}(\mathrm{~m} / \mathrm{s})$ & $30.6 \times \cos 25^{\circ}$ & 31.3 & 31.1 & 1.4 \\
& $V_{y}(\mathrm{~m} / \mathrm{s})$ & $30.6 \times \sin 25^{\circ}$ & 4.3 & 4.5 & -109.0 \\
& $\Omega_{z}(\mathrm{deg} / \mathrm{s})$ & 0 & -89.9 & -95.3 & - \\
\hline & $\Omega_{x}(\mathrm{deg} / \mathrm{s})$ & 0 & -13.2 & -15.8 &
\end{tabular}

scheduled, the vehicle simply spins out and skids sideways after the impact, eventually with a lateral acceleration of approximately $0.7 \mathrm{~g}$.

Then the same collision problem is solved by both the proposed approach based on the 4-DOF vehicle model and the momentum-conservation method as formulated in Section 2. For easier comparison, the obtained post-impact target vehicle states are collected in Table 2 under the headings '4-DOF' and 'planar' separately.

With the proposed approach, the post-impact translational velocities agree well with those computed by CarSim. The predicted yaw rate and roll rate show certain deviations; however, compared with the results obtained from the planar model, the accuracy has been substantially improved. The error in roll rate prediction is largely due to the non-linear effects of the suspensions. The pre- and post-impact translational and rotational velocities are also plotted on Figure 7 and marked with circles.
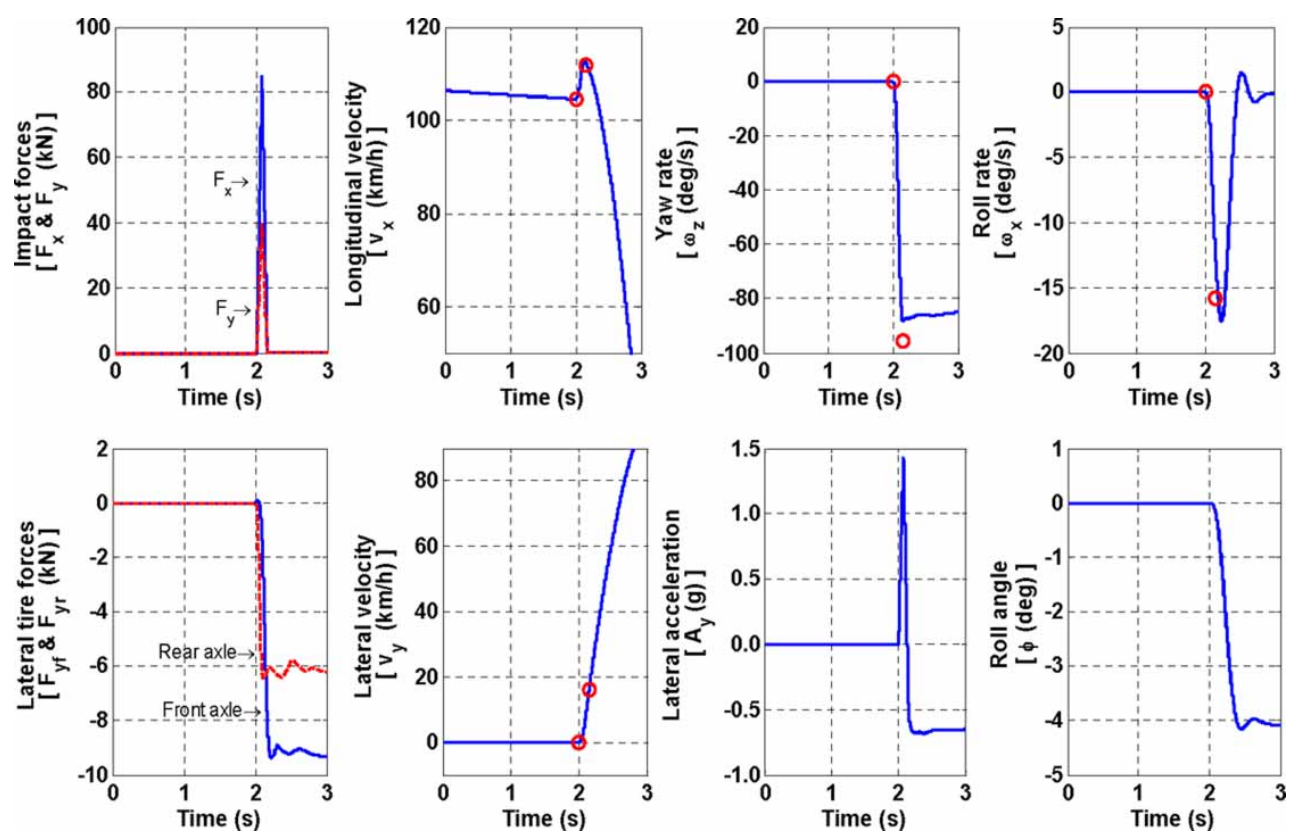

Figure 7. Responses of the target vehicle involved in a collision. Circles indicate the pre-impact and the post-impact vehicle states predicted by the proposed approach. 

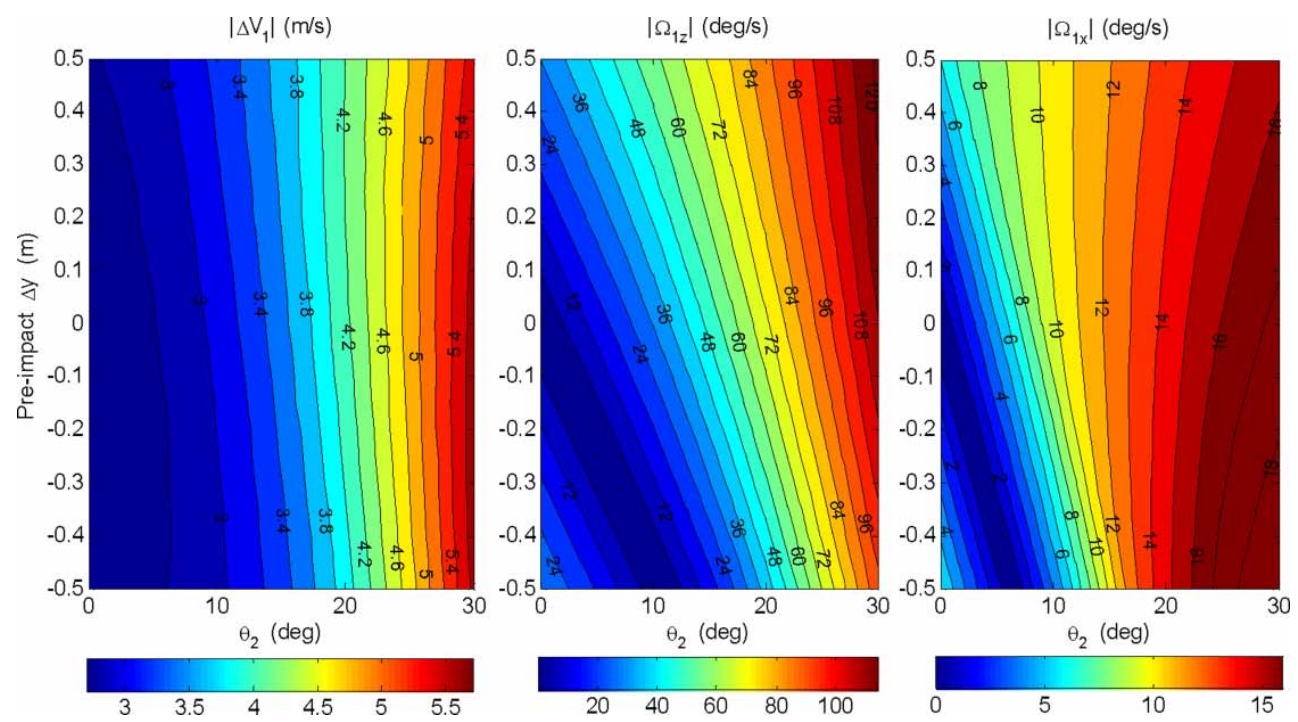

Figure 8. Contour plots of absolute CG velocity change, post-impact yaw rate, and roll rate for the target vehicle in angled rear-end collisions.

The momentum-conservation-based planar model over-predicts the yaw rate, and cannot be used to calculate the vehicle roll motion. As a matter of fact, if we further analyse the CarSim results within the collision time interval, it is found that the ratio of the impulses due to tyre lateral forces and those due to the external impact is roughly 0.37 for this particular case, a value too substantial to be disregarded. By ignoring the contribution of tyre forces in a collision, the momentum-conservation computation risks producing significant errors in predicted results.

Figure 8 shows contour plots for a matrix of rear-end collisions, with respect to absolute $\mathrm{CG}$ velocity change, post-impact yaw rate, and roll rate for the target vehicle. The simulated rear-end collisions are similar to that illustrated in Figure 6, but the incidence angle of the bullet vehicle and the impact point on the target vehicle are varied. Prior to the impact, the target vehicle was parallel to the road tangent, and the bullet vehicle had an orientation angle $\left(\theta_{2}\right)$ between $0^{\circ}$ and $30^{\circ}$, whereas the collision offset $(\Delta y)$ varies between $-0.5 \mathrm{~m}$ and $0.5 \mathrm{~m}$. Both vehicles were travelling along their own longitudinal axes, with velocities $v_{1 x}=29 \mathrm{~m} / \mathrm{s}$ and $v_{2 x^{\prime}}=33.5 \mathrm{~m} / \mathrm{s}$. Uniform values of collision coefficients are assumed for all cases: $e=0.20$, $\mu=0$, thus $\Gamma=\theta_{2}$.

Given the $4.5 \mathrm{~m} / \mathrm{s}$ pre-impact closing velocity, Figure 8 shows that the post-impact yaw rate of the target vehicle can exceed $100^{\circ} / \mathrm{s}$ when the incidence angle is just $30^{\circ}$. Overall the post-impact yaw rate and roll rate are much more sensitive to incidence angle than to collision offset. Similar contour plots can also be plotted for collisions of other configurations, such as side impact and frontal impact. These plots offer an efficient and a reasonably accurate way to examine the consequences of a class of light collisions.

In brief, in this section a collision problem is first solved in a full-feature vehicle dynamics software, and the computational results are used to evaluate the accuracy of other simplified approaches. The approach proposed in this study is based on a 4-DOF vehicle model and accounts for impact forces and tyre forces simultaneously. Computational results confirm improved accuracy in the predicted post-impact vehicle states, especially translational velocities and roll rate. 


\section{Conclusions}

Vehicle collision problems have been approached in diverse disciplines. The focus of this study is on the characterisation of changes in vehicle kinematic states due to light impacts, including translational velocities, yaw rate, and roll rate. The proposed vehicle impact model extends the traditional momentum-conservation approach by incorporating tyre forces and sprung mass roll motion. Numerical results demonstrate improved accuracy in predicting post-impact vehicle states. The proposed approach provides an efficient and reasonably accurate way to characterise vehicle motions immediately after an impact. The developed collision model is useful for the prediction of post-impact vehicle motions and the development of enhanced vehicle safety systems.

\section{References}

[1] V. Babu, K.R. Thomson, and C. Sakatis, LS-DYNA 3D interface component analysis to predict FMVSS 208 occupant responses, SAE Tech. Paper 2003-01-1294, 2003.

[2] K. Solanki, D.L. Oglesby, C.L. Burton, H. Fang and M.F. Horstemeyer, Crashworthiness simulations comparing PAM-CRASH and LS-DYNA, SAE Tech. Paper 2004-01-1174, 2004.

[3] T.D. Day, An overview of the EDSMAC4 collision simulation model, SAE Tech. Paper 1999-01-0102, 1999.

[4] H. Steffan and A. Moser, The collision and trajectory models of PC-CRASH, SAE Tech. Paper 960886, 1996.

[5] R. McHenry, Development of a computer program to aid the investigation of highway accidents, Calspan Rep. No. VJ-2979-V-1, DOT HS-800 821, 1971.

[6] R.M. Brach, Mechanical Impact Dynamics: Rigid Body Collisions, Wiley, New York, 1991.

[7] R.M. Brach and R.M. Brach, Vehicle Accident Analysis and Reconstruction Methods, SAE, Warrendale, PA, 2005.

[8] $\longrightarrow$, Crush energy and planar impact mechanics for accident reconstruction, SAE Tech. Paper 980025, 1998.

[9] A.L. Cipriani, F.P. Bayan, M.L. Woodhouse, A.D. Cornetto, A.P. Dalton, C.B. Tanner, T.A. Timbario and E.S. Deyerl, Low speed collinear impact severity, a comparison between full scale testing and analytical prediction tools with restitution analysis, SAE Tech. Paper 2002-01-0540, 2002.

[10] J.W. Cannon, Dependence of a coefficient of restitution on geometry for high speed vehicle collisions, SAE Tech. Paper 2001-01-0892, 2001.

[11] V.W. Antonetti, Estimating the coefficient of restitution of vehicle-to-vehicle bumper impacts, SAE Tech. Paper 980552, 1998.

[12] R.M. Brach, Modeling of low-speed, front-to-rear vehicle impacts, SAE Tech. Paper 2003-01-0491, 2003.

[13] K.L. Monson and G.J. Germane, Determination and mechanisms of motor vehicle structural restitution from crash test data, SAE Tech. Paper 1999-01-0097, 1999.

[14] M.C. Marine, On the concept of inter-vehicle friction and its application in automobile accident reconstruction, SAE Tech. Paper 2007-01-0744, 2007.

[15] L. Segel, Theoretical prediction and experimental substantiation of the response of the automobile to steering control, Proceedings of Institute of Mechnical Engineers, pp. 310-330, 1956.

[16] R.D. Anderson, J.B. Welcher, T.J. Szabo, J.J. Eubanks and W.R. Haight, Effect of braking on human occupant and vehicle kinematics in low speed rear-end collisions, SAE Tech. Paper 980298, 1998.

[17] M. Huang, Vehicle Crash Mechanics, CRC Press, Boca Raton, FL, 2000.

[18] M.S. Varat and S.E. Husher, Vehicle impact response analysis through the use of accelerometer data, SAE Tech. Paper 2000-01-0850, 2000.

[19] Mechanical Simulation Corporation, Ann Arbor, MI, 2006. CarSim Homepage available at http://www.carsim.com.

\section{Appendix}

$$
A_{11}=\left(\begin{array}{cccc}
m_{1} & 0 & -m_{1} \frac{\Delta t}{2} V_{1 y} & 0 \\
0 & m_{1}+\frac{\Delta t}{2} \frac{C_{f 1}+C_{r 1}}{V_{1 x}} & m_{1} \frac{\Delta t}{2} V_{1 x}+\frac{\Delta t}{2} \frac{a_{1} C_{f 1}-b_{1} C_{r 1}}{V_{1 x}} & -m_{R 1} h_{1} \\
0 & \frac{\Delta t}{2} \cdot \frac{-a_{1} C_{f}+b_{1} C_{r}}{V_{1 x}} & -I_{z z 1}-\frac{\Delta t}{2} \frac{a_{1}^{2} C_{f 1}+b_{1}^{2} C_{r 1}}{V_{1 x}} & -I_{x z 1} \\
0 & -m_{R 1} h_{1} & I_{x z 1}-m_{R 1} h_{1} \frac{\Delta t}{2} V_{1 x} & I_{x x s 1}+\frac{\Delta t}{2} D_{s 1}
\end{array}\right),
$$




$$
\begin{aligned}
& A_{13}=\left(\begin{array}{cccc}
-1 & 0 & 0 & 0 \\
0 & -1 & 0 & 0 \\
-y_{A} & x_{A} & 0 & 0 \\
0 & -\left(z_{A}-h_{1}\right) & 0 & 0
\end{array}\right) \\
& A_{22}=\left(\begin{array}{cccc}
m_{2} & 0 & -m_{2} \frac{\Delta t}{2} V_{2 y^{\prime}} & 0 \\
0 & m_{2}+\frac{\Delta t}{2} \frac{C_{f 2}+C_{r 2}}{V_{2 x^{\prime}}} & m_{2} \frac{\Delta t}{2} V_{2 x^{\prime}}+\frac{\Delta t}{2} \frac{a_{2} C_{f 2}-b_{2} C_{r 2}}{V_{2 x^{\prime}}} & -m_{R 2} h_{2} \\
0 & \frac{\Delta t}{2} \cdot \frac{-a_{2} C_{f 2}+b_{2} C_{r 2}}{V_{2 x^{\prime}}} & -I_{z z 2}-\frac{\Delta t}{2} \frac{a_{2}^{2} C_{f 2}+b_{2}^{2} C_{r 2}}{V_{2 x^{\prime}}} & -I_{x z 2} \\
0 & -m_{R 2} h_{2} & I_{x z 2}-m_{R 2} h_{2} \frac{\Delta t}{2} V_{2 x^{\prime}} & I_{x x s 2}+\frac{\Delta t}{2} D_{s 2}
\end{array}\right), \\
& A_{23}=\left(\begin{array}{cccc}
0 & 0 & -1 & 0 \\
0 & 0 & 0 & -1 \\
0 & 0 & -y_{A^{\prime}} & x_{A^{\prime}} \\
0 & 0 & 0 & -\left(z_{A^{\prime}}-h_{2}\right)
\end{array}\right), \quad A_{31}=\left(\begin{array}{cccc}
0 & 0 & 0 & 0 \\
0 & 0 & 0 & 0 \\
0 & 0 & 0 & 0 \\
\cos \Gamma & \sin \Gamma & d_{c} \cos \Gamma-d_{d} \sin \Gamma & 0
\end{array}\right) \text {, } \\
& A_{33}=\left(\begin{array}{cccc}
1 & 0 & \cos \theta_{2} & -\sin \theta_{2} \\
0 & 1 & \sin \theta_{2} & \cos \theta_{2} \\
\mu \cos \Gamma+\sin \Gamma & \mu \sin \Gamma-\cos \Gamma & 0 & 0 \\
0 & 0 & 0 & 0
\end{array}\right) \\
& A_{32}=\left(\begin{array}{cccc}
0 & 0 & 0 & 0 \\
0 & 0 & 0 & 0 \\
0 & 0 & 0 & 0 \\
-\sin \theta_{2} \sin \Gamma-\cos \theta_{2} \cos \Gamma & \sin \theta_{2} \cos \Gamma-\cos \theta_{2} \sin \Gamma & d_{a} \cos \Gamma-d_{b} \sin \Gamma & 0
\end{array}\right) \\
& B=\left(\begin{array}{llllllllllll}
m_{1} v_{1 x} & 0 & 0 & 0 & m_{2} v_{2 x^{\prime}} & 0 & 0 & 0 & 0 & 0 & 0 & B_{12}
\end{array}\right)^{\prime}
\end{aligned}
$$

where $B_{12}=-e\left[\left(-v_{2 x^{\prime}} \sin \theta_{2}\right) \cdot \sin \Gamma+\left(v_{1 x}-v_{2 x^{\prime}} \cos \theta_{2}\right) \cdot \cos \Gamma\right]$. 
